\title{
Two-port network Analysis and Modeling of a Balanced Armature Receiver
}

\author{
Noori Kim ${ }^{\mathrm{a}}$, Jont Allen ${ }^{\mathrm{a}, *}$
}

${ }^{a}$ Department of Electrical and Computer Engineering, University of Illinois at Urbana-Champaign, 1206 W. Green Street, Urbana, IL 61801, USA

\section{Abstract}

Models for acoustic transducers, such as loudspeakers, mastoid bone-drivers, hearing-aid receivers, etc., are critical to many acoustic applications. Acoustic transducers employ two-port models to convert between acoustic and electromagnetic signals. This study analyzes a widely-used commercial hearing-aid receiver ED series, manufactured by Knowles Electronics, Inc., as well as their SPICE circuit model simulation. The analysis has shown that these models can be greatly improved and simplified. Electromagnetic transducer modeling must consider two key elements: a semiinductor and a gyrator. The semi-inductor accounts for electromagnetic eddy-currents, the 'skin effect' of a conductor, (Vanderkooy, 1989), while the gyrator (McMillan, 1946; Tellegen, 1948) accounts for the anti-reciprocity characteristic [Lenz's law (Hunt, 1954, p. 113)]. Aside from Hunt (1954), few publications have included the gyrator element in their electromagnetic transducer models. The most prevalent method of transducer modeling evokes the mobility method, using an ideal transformer instead of a gyrator (Beranek, 1954). Without taking the anti-reciprocity into account, this approach overly complicates the analysis. The present study proposes a novel, simplified and rigorous receiver model. Hunt's two-port parameters, the electrical impedance $Z_{e}(s)$, acoustic impedance $Z_{a}(s)$ and electro-acoustic transduction coefficient $T_{a}(s)$, are calculated by using ABCD and impedance matrix methods (Van Valkenburg, 1964). The results from electrical input impedance measurements $Z_{\text {in }}(s)$, which vary with given acoustical loads, are used in the calculation (Weece and Allen, 2010). The hearing-aid receiver transducer model is designed based on energy transformation flow [electric $\rightarrow$ mechanic $\rightarrow$ acoustic]. The model has been verified with electrical input impedance, laser, vacuum, and pressure experiments. This receiver model is suitable for designing most electromagnetic transducers, and it can ultimately improve the design of hearing-aid devices, providing a much more simplified analysis.

Keywords: , Hearing-aid receiver, Balanced armature receiver modeling

\section{Introduction}

A transducer converts energy from one modality to another. A hearing-aid receiver is an electromagnetic loudspeaker that converts an electrical signal to acoustical pressure. It is referred to as an electromagnetic transducer, because small magnets are involved. Since these miniature loudspeakers are widely used in modern hearing-aids, and remain one of the most expensive components of the hearing-aids, a detailed understanding of them is critical.

There are two elements of special interest in the electromagnetic transducer: the semi-inductor and the gyrator. The semi-inductor component is required to account for eddy-current diffusion (the 'skin effect'). In 1989, Vanderkooy demonstrated that, at high frequencies, the behavior of the impedance of a loudspeaker changes from the behavior of a normal inductor to that of a semi-inductor, because of the eddy current diffusing into the iron pole structure of the loudspeaker. Using a Bessel function ratio, Warren and LoPresti (2006) represented Vanderkooy's semi-inductor model as a 'diffusion ladder network,' a combination of resistors and inductors. In 2010, Weece and Allen used this representation in a bone driver model. After demagnetized the bone driver, they established the $\sqrt{s}$ behavior, and determined the ladder network elements from the measured electrical impedance of the transducer. Thorborg et al. (2007) also introduced a loudspeaker model with lumped circuit elements including a semi-inductor.

In 1946, McMillan introduced the gyrator component as a network element. Two years later, Tellegen (1948) coined the term 'gyrator,' and categorized it as a fifth network element, along with the capacitor, resistor, inductor, and ideal transformer. Other than Hunt's 1954 publication, few papers have implemented anti-reciprocity in their

${ }^{*}$ Corresponding author. 2061 Beckman Institute, 405 N. Mathews, Urbana, IL 61801, USA. Tel. (+1) 217-369-7711. 
electromagnetic transducer model, with a gyrator,

$$
\left[\begin{array}{l}
E(\omega) \\
F(\omega)
\end{array}\right]=\left[\begin{array}{cc}
Z_{e}(s) & -T(s) \\
T(s) & z_{m}(s)
\end{array}\right]\left[\begin{array}{l}
I(\omega) \\
V(\omega)
\end{array}\right]
$$

which converts electrical signals to mechanical force, where $E$ is the voltage, $I$ is the current, $F$ is the force and $V$ is the particle velocity. The direction of $I$ and $V$ is defined as into the network. This matrix involves an electrical impedance $Z_{e}=\left.\frac{E}{I}\right|_{V=0}$, a mechanical impedance $z_{m}=\left.\frac{F}{V}\right|_{I=0}$, and an electromagnetic transduction coefficient $T=\left.\frac{F}{I}\right|_{V=0}=-\left.\frac{E}{V}\right|_{I=0}$. We call $Z_{e}, z_{m}$, and $T$ the Hunt parameters ${ }^{1}$, which are functions of the Laplace (complex) frequency $s=\sigma+j \omega$. When the two off-diagonal elements of Eq. 1 are equal, the system is reciprocal; if they are opposite in sign, the system is anti-reciprocal, which is a necessary condition of the electromagnetic transducer. Equation 1 may be 'factored' as an ABCD (transmission) matrix, as shown in Fig. 1.

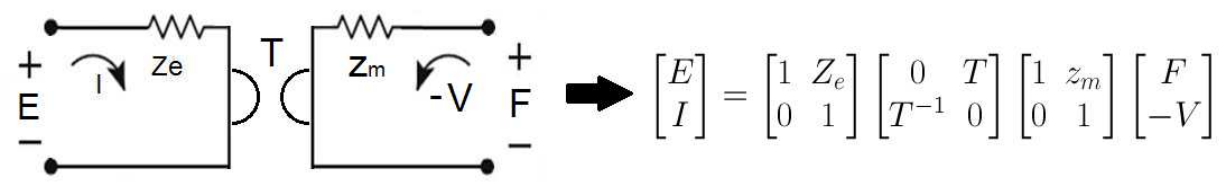

Figure 1: Schematic representation of an electro-magnetic transducer and the equivalent the ABCD matrix representation. The electrical and mechanical sections are coupled using the gyrator.

The impedance matrix is used when making measurements. For instance, system's electrical input impedance and output acoustic impedance (or output mechanical impedance) can be represented with the impedance matrix elements, $Z_{e}$ and $Z_{a}$ (or $z_{m}$ ). The ABCD matrix representation is useful for network modeling, and then is transformed to an impedance matrix.

One may convert from an impedance matrix to an $\mathrm{ABCD}$ matrix using the relationship:

$$
\left[\begin{array}{cc}
A & B \\
C & D
\end{array}\right]=\frac{1}{T_{a}}\left[\begin{array}{cc}
Z_{e} & \Delta_{Z} \\
1 & Z_{a}
\end{array}\right],
$$

where $\Delta_{Z}=Z_{e} Z_{a}+T_{a}^{2}$, and $T_{a}$ is the acoustic transduction impedance (Van Valkenburg, 1964).

Inverting the acoustical and electrical variables in Eq. 4 gives the inverse $\mathrm{ABCD}$ system:

$$
\left[\begin{array}{l}
P(\omega) \\
U(\omega)
\end{array}\right]=\frac{1}{\Delta_{A B C D}}\left[\begin{array}{cc}
-D(s) & B(s) \\
C(s) & A(s)
\end{array}\right]\left[\begin{array}{c}
E(\omega) \\
I(\omega)
\end{array}\right],
$$

where $\Delta_{A B C D}=D A+B C=-1$. Equation 3 allows one to calculate the Thevenin pressure or Norton velocity in terms of the electrical input.

The electromagnetic transducer models from both Weece and Allen (2010) and Knud (2007), an ideal transformer was used to convert electrical current into mechanical force (or acoustical pressure) of the transducer. As described in Beranek (1954), the mobility strategy (along with the impedance or admittance analogy) is appropriate to represent electrical to mechanical transduction when designing electromagnetic transducers using an ideal transformer. However, this method that employs a transformer instead of a gyrator fails to provide an intuitive explanation of the 'antireciprocity' characteristic of the electromagnetic transducer, which follows from Maxwell-Faraday's law and Lenz's law. Including the gyrator in transducer models allows for an instinctive interpretation of physical properties. For example, with of a gyrator representing the mechanical and electrical transformation, one may simply represent a stiffness as a capacitor, and mass as an inductor, in series combination. Given the mobility (dual) network, one must swap the inductor and capacitor, and place them in parallel combination. The dual network is not intuitive.

\footnotetext{
${ }^{1}$ Wegel (1921) was the first to define Eg. 1 in this form, so perhaps we should call these the Wegel parameters.
} 


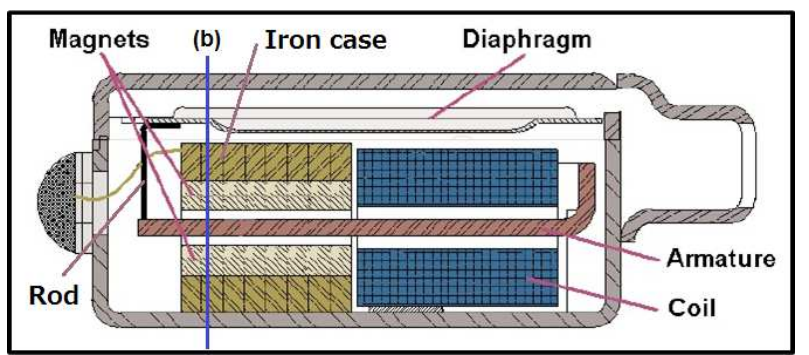

(a) The structure of ED7045 receiver

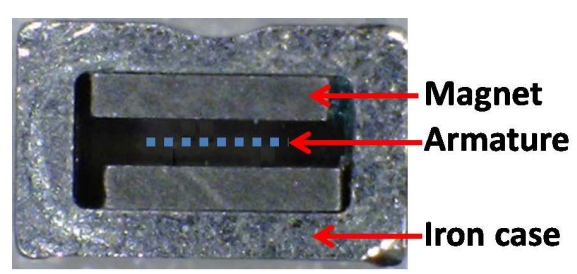

(b) The cross-section view of the receiver

Figure 2: (a): A schematic of a BAR. An electrical current in the coil comes from the transducer's electrical input terminals; the current induces a magnetic field to move the armature. (Modified from Knowles documentation about ED receiver series.) Note that the port location of ED7045 receiver is rotated $90^{\circ}$ to longer side, which is different from this general scheme of ED series. (b): The picture of the BAR crossed by line (b) in figure (a). There is a space for the armature to vibrate (vertically) between the magnets. Magnets are sandwiching the armature (a blue, dotted line). A laminated iron case surrounds the magnets and the armature.

The Knowles Electronics ${ }^{2}$ ED series receivers, including the ED7045 and ED1913, are balanced armature receivers (BAR) widely used as hearing-aid receivers. These receivers consist of a coil, armature, magnets, and a diaphragm, s shown in Fig. 2(a). Unlike the traditional moving-coil drivers, the coil of the balanced armature driver has a fixed sition and does not move (Jensen et al., 2011), thereby reducing the mass and providing more space for a longer coil.

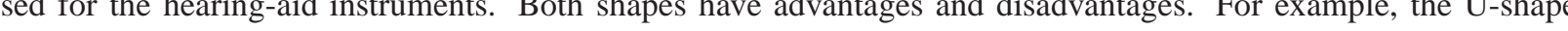
accounts for lowering vibration of the body. Two permanent magnets sandwich the armature, and the magnets and the coil are next to each other. The armature is placed through the center of the coil, without touching or connecting to it, and the movement of the armature is directly related to the vibration of the diaphragm through a thin rod (Fig. 2 (a)). The ED receiver is $6.32 \mathrm{~mm} \times 4.31 \mathrm{~mm} \times 2.97 \mathrm{~mm}$ in size.

\section{Sensitivity analysis of ED series SPICE model}

Figure 3 shows the Knowles Electronics commercial SPICE circuit model.(Killion, 1992) Not all of the shown components are physically present in the receiver; rather, they represent various physical and acoustic properties, such as cavities, tubes, and stiffness.

From Fig. 3, this SPICE model of the receiver contains a gyrator. However, there are not enough components to support the electrical part of the transducer on the left side of the gyrator. To represent the electrical circuit property, the inductance of the coil must accompany the resistance. Furthermore, each inductor must include a semi-inductor to account for the magnetic diffusion ((Vanderkooy, 1989)). The semi-capacitor (CMAG) series on the right side of the gyrator should be on the left side, as a parallel combination of an inductor and semi-inductor (Thorborg et al., 2007), consistent with the impedance behavior of the semi-inductor mainly affecting the high frequency response.

In order to fully understand each component on the system, we implemented the Knowles PSpice model in Matlab using transmission matrices. Unlike PSpice, Matlab provides a much better platform for model manipulation, which does not critically depend on the user's operating system (Knowles' PSpice model is directly tied to both the Cadence Orcad Schematics and Capture, and Windows XP).

We then performed a sensitivity analysis by changing each component value by $+/-10 \%$ and found that the output changed by less than $0.001 \%$, within the frequency range of $0.1-20[\mathrm{kHz}]$. From this analysis, we concluded that many of the elements, as labeled, have a truly negligible effect on either the electrical or acoustical input impedance, or on the transfer function (Fig. 4). The most critical result of this analysis is that the semi-capacitor in the PSpice model is one of the 'insensitive' components, and thus the model, Fig. 3, can be significantly simplified. This discovery further motivated our object, to design a simplified and rigorous BAR model.

\footnotetext{
${ }^{2}$ Knowles Electronics, Itasca, IL (http://www.knowles.com)
} 


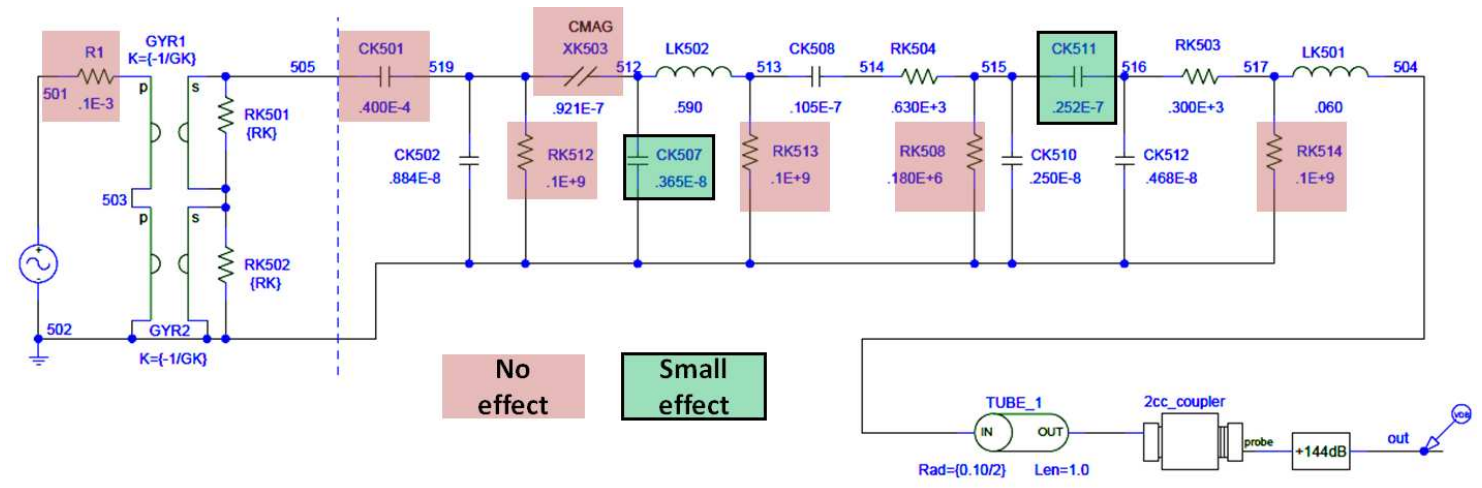

Figure 3: PSpice model of the Knowles ED receiver series. The refined circuit spice model of ED receiver by reducing 'no effect' components. The 'no effect' components are marked in red. The 'small effect'components are marked in green. The 'no-effect' resistors were added to maintain DC stability of PSpice.
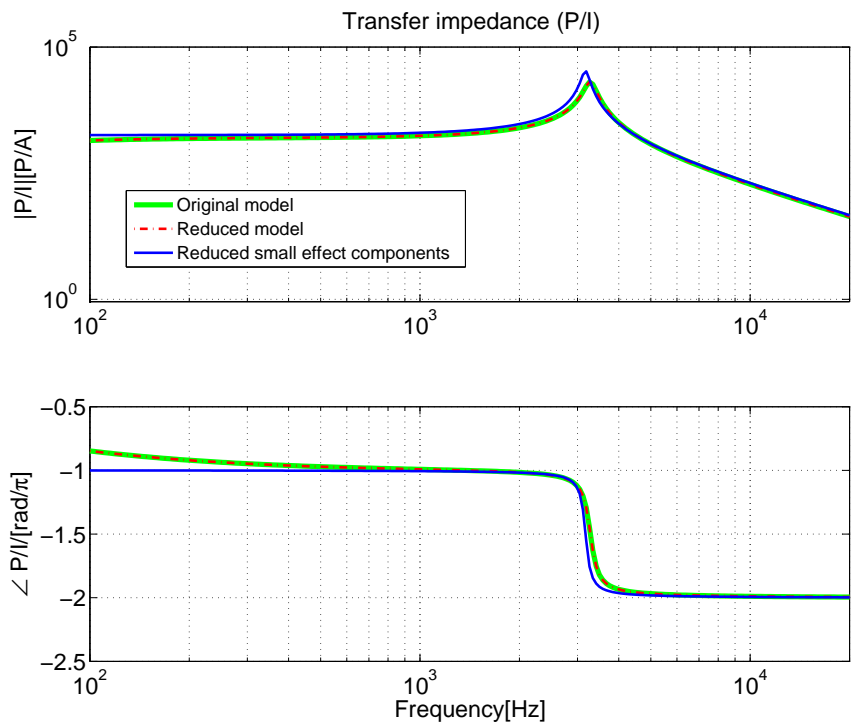

Figure 4: The simulated transfer function: Sensitivity analysis based on Fig. 3 was performed by adding or removing each component. Removing 'no-effect' components does not change the original frequency response. The 'original model' and the 'reduced model' conditions are marked with a thick green line and a dashed red line, respectively. Both conditions are on top of each other. Deleting small-effect components ('Reduced small effect components') only shows a slight difference from the original response which is plotted in blue. 
The remainder of this paper is structured as follows: Section 2 introduces the theoretical concepts that are specifically related to designing electro-magnetic transducer models. Section 3 presents the experimental methods used in the present study. Section 4 includes the results from the experiments. Hunt's parameters are derived via electrical impedance measurements to establish the fundamental properties of the transducers. We present the proposed transducer model in Section 5, which is followed by a model verification via experimental comparison in Section 6.

\section{Theories}

\subsection{Two-port network theory}

Two-port networks (Eq. 1) generalize the one-port Thevenin/Norton model, are based on Ohm's law, and are used to characterize linear transducers (Hunt, 1954; Van Valkenburg, 1964; Wegel, 1921).

Carlin and Giordano (1964) identified six properties of two-port networks: 1. Linearity - A network is linear if the scaling and addition of the input causes the same effect on the output. 2. Time-Invariance - In a time-invariant network, shifting a signal in time does not affect the characteristics of the network. 3. Passivity - A network is passive if its components do not contain a power source. 4. Causality - A causal network's output does not predict the input. 5. Real-Time Function - A network must respond to a real time-signal with a real output response. 6. Reciprocity - A two-port network is reciprocal if and only if the off-diagonal elements of the impedance matrix are equal.

Based on McMillan (1946) and Hunt (1954), a moving-armature electromagnetic transducer can be approximately considered to a linear, time-invariant, passive, causal, real-time and anti-reciprocal system (the off diagonal elements if Eq. 1 have opposite sign). Note that a semi-inductor impedance $\sqrt{s}$ is linear, since it can be transformed into the time domain response via the inverse Laplace transform (a linear causal operator) according to

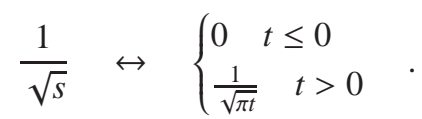

\subsection{Two-port relationship: Impedance and $A B C D$ (Transmission) matrices}

The two-port impedance a matrix representation (Eq. 1) is matrix extension of Ohm's law for the 'electro-mechanical' system. The two-port 'electro-acoustic' transducer equation can alternatively be represented in ABCD matrix form, as given by Eq. 4. The fundamental difference of the two matrix systems lies in the coupling of the 'electro-acoustic' transducer, between the mechanical and the acoustic signals (i.e., $\pm \mathrm{T}(\mathrm{s})$ ). Specifically, the electrical input parameters $E$ and $I$ on the left side of the network are expressed in terms of the acoustical variables, the pressure $P$ and the volume velocity $U$, on the right side of the network (or alternatively $F$ and $V$ for an 'electro-mechanical' transducer), via the four frequency dependant parameters A, B, C, and D:

$$
\left[\begin{array}{c}
E(\omega) \\
I(\omega)
\end{array}\right]=\left[\begin{array}{ll}
A(s) & B(s) \\
C(s) & D(s)
\end{array}\right]\left[\begin{array}{c}
P(\omega) \\
-U(\omega)
\end{array}\right]
$$

As shown in Fig. 1, each network element may be represented with a 2 by 2 ABCD matrix, with the volume velocity $U$ defined as flowing out of the element (here the '-' sign). Thus multiple elements' matrices can be 'chained' (i.e., factored) in accordance with different combinations of the elements (i.e., series or shunt). This allows one to represent the network analysis using matrix multiplication. Therefore, when we transform the ABCD matrix to an impedance matrix, it is necessary to force a negative sign for the volume velocity to maintain consistency.

Based on Eq. 4, we can define 'open circuit' and 'short circuit' conditions for both the acoustical and the electrical impedance cases. Thus, the acoustical impedance $\left(Z_{a}\right)$ and electrical impedance $\left(Z_{e}\right)$ can be defined as:

$$
\begin{aligned}
& Z_{a}(s) \equiv \frac{P(\omega)}{U(\omega)}=\left.\frac{D(s)}{C(s)}\right|_{I=0}=\left.\frac{B(s)}{A(s)}\right|_{E=0} \\
& Z_{e}(s) \equiv \frac{E(\omega)}{I(\omega)}=\left.\frac{A(s)}{C(s)}\right|_{U=0}=\left.\frac{B(s)}{D(s)}\right|_{P=0} .
\end{aligned}
$$

As described earlier, the corresponding Hunt parameters, $Z_{e}$ and $Z_{a}$ for acoustics (or alternatively $z_{m}$ for mechanics), are defined under the open circuit condition, $I=0$ and $U$ (or $V)=0$, respectively. The specific methods and results are discussed in Section 3 and 4. 


\subsection{Gyrator and anti-reciprocity}

When electric fields $(\bar{E})$ induce magnetic flux $\left(\Phi=\bar{B} S_{a}\right.$ ), the relationship between those two fields (considering the field area $S_{a}$ ) is described by the Faraday-Lenz law,

$$
\underbrace{\oint_{C} \bar{E} d l}_{\text {voltage } E}=-\frac{d}{d t} \int_{S_{a}} \bar{B} d A .
$$

This equation says that the induced voltage is equal to the negative rate of change of the total flux. Note that the minus sign comes from the Lenz's law due to conservation of energy.

A gyrator is required when quantifying the anti-reciprocal coupling between the electric and the magnetic fields (Tellegen, 1948), as shown in Fig. 1 (Hunt (1954)). The impedance matrix of a gyrator is

$$
\left[\begin{array}{l}
E \\
F
\end{array}\right]=\left[\begin{array}{cc}
0 & -T \\
T & 0
\end{array}\right]\left[\begin{array}{l}
I \\
V
\end{array}\right],
$$

and the corresponding $\mathrm{ABCD}$ matrix relationship is

$$
\left[\begin{array}{c}
E \\
I
\end{array}\right]=\left[\begin{array}{cc}
0 & T \\
1 / T & 0
\end{array}\right]\left[\begin{array}{c}
F \\
-V
\end{array}\right] .
$$

The gyration coefficient $T$ is one of the three Hunt parameters of Eq. 1. In an electromagnetic transducer, a gyrator (Fig. 8a) converts the potential on the electrical side to a flow on the mechanical side. Likewise, from Eq. 8b, the potential (force or voltage) in one side becomes the flow (velocity or current) on the other side at the gyrator. Finally, cascading two ideal gyrators act as an ideal transformer

$$
\left[\begin{array}{cc}
0 & T \\
1 / T & 0
\end{array}\right]\left[\begin{array}{cc}
0 & T \\
1 / T & 0
\end{array}\right]=\left[\begin{array}{ll}
1 & 0 \\
0 & 1
\end{array}\right] .
$$

To account for the mechanic-acoustic transition in electro-acoustic transducers, the force and particle velocity in the mechanical part are transferred into the pressure $P$ and the volume velocity $U$ of the acoustical part. In a physical sense, the diaphragm or speaker cone vibration determines the air particle movements associated with volume, which is the source of the radiated pressure and the loaded impedance. Because of this radiation, the mechanical delay $T$ must be augmented by an acoustic propagation delay in the electro-acoustic circuit to represent the complete relation of the system,

$$
T_{a}(s)=T e^{-s \tau_{0}} .
$$

Note that $T_{a}$ represents an entire transit relationship of the electro-acoustic transducers, from electric to mechanic to acoustic, including a system delay $\left(\tau_{0}\right)$, whereas the gyration coefficient $T$ only considers the local transition from the electric to the mechanical.

In the case of a moving-coil loudspeaker, $T$ is defined as a constant $B_{0} l$, the product of the magnetic field $\left(B_{0}\right)$ and the length of the wire coil (l) (Dodd et al. (2004); Thorborg et al. (2007)), which follows from the Lorentz force (or Laplace force),

$$
F=I l \times \bar{B},
$$

where $F$ is the net force on a stationary wire with a steady current $I$, and $l$ is the length of wire.

\section{An alternative theory for $T$}

Following Hunt's 1954 chapter 7, Jensen et al. (2011) designed a non-linear time-domain BAR model, and found $T$. Based on their theory, the input force of the moving-armature transducer system employs 'the tractive force' which tries to minimize the air gap between the armature and the magnet;

$$
F=\frac{S_{a} \bar{B}^{2}}{2 \mu_{0}}=\frac{\Phi_{0}^{2}}{2 \mu_{0} S_{a}},
$$


where $\bar{B}$ is the magnetic field across the air gap $\left[\mathrm{Wb} / \mathrm{m}^{2}\right], S_{a}$ is the transverse area of the armature with the permanent magnet $\left[\mathrm{m}^{2}\right], \mu_{0}$ is the permeability in free space $\left(4 \pi 10^{-7}[\mathrm{H} / \mathrm{m}]\right)$, and $\Phi_{0}$ is the total magnetic flux in the air gap [Wb] (Hunt, 1954; Jensen et al., 2011).

Jensen et al. defined the nonlinear time-domain representation of the transduction coefficient $T$ (Jensen et al. (2011) Eq. 4). In the frequency domain, if the input signal is small (small armature displacement, $x<<D$ ), Eq. ?? reduces to the constant,

$$
T \approx-\left.\frac{2 \mu_{o} n S_{a} F_{M}}{D^{2}}\right|_{I=0}
$$

where $F_{M}$ is the magnetomotive force of the permanent magnet [A.turns], $n$ is the number of the turns of coil winding [turns], and $D$ is the initial air gap distance between armature and the magnet [m].

The Eq. 12 comes from the diagonal magnetic term of the Maxwell stress tensor $\sigma$ (Sommerfeld, 1964), which is derived from the Lorentz force density $f\left[\mathrm{~N} / \mathrm{m}^{3}\right]$,

$$
\nabla \cdot \sigma=f+\epsilon_{0} \mu_{0} \frac{\partial \bar{S}}{\partial t}
$$

where $\epsilon_{0}$ is the vacuum permittivity $\left(8.85 \cdot 10^{-12}[\mathrm{~F} / \mathrm{m}]\right)$ and $\bar{S}$ is the Poynting vector $\bar{S}=\frac{1}{\mu_{0}} \bar{E} \times \bar{B}$. Therefore $T$ in Eq. 13 should be constant to $B_{0} l$.

\subsection{Semi-inductor}

Vanderkooy (1989) first identified the electrical impedance representation of the semi-inductor as proportional to $\sqrt{s}$ due to the eddy current. A simple impedance formula of the semi-inductor is derived with the assumption that the length of a coil sheet is infinite. Neglecting the radius of the coil and the gap between the magnetic material and the wire,

$$
Z_{\text {semi }}=n^{2} \sqrt{\frac{\mu s}{\sigma}}=K \sqrt{s}
$$

where $K$ is semi-inductance per unit length in semi-henrys, $n$ is the number of coil winding turns of wire, $\mu$ is the permeability, and and $\sigma$ is the conductivity of the iron armature.

Semi-inductors which result from magnetic diffusion are not commonly found in circuit analysis. However, it is a key element in characterizing the 'eddy-current' in electromagnetic models, such as loudspeakers or bone drivers. In a BAR, the eddy current is distributed through the surface of the armature, as well as on the cross section of the laminated iron box which surrounds the magnets (Fig. 2). In a dynamic loudspeaker, on the other hand, the coil is directly connected to the diaphragm and the eddy-current is distributed through the surface of an iron core (a polepiece structure).

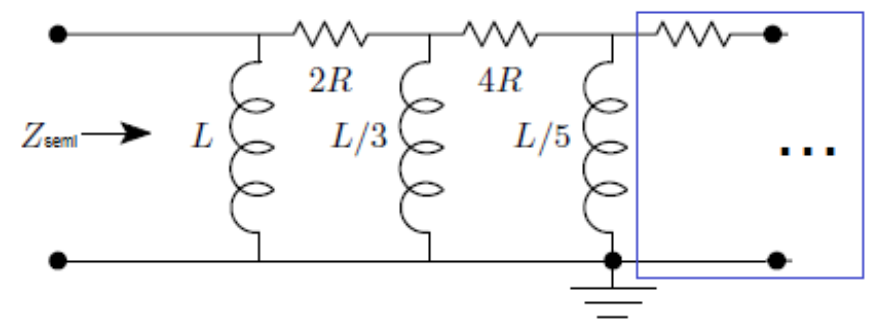

Figure 5: Semi-inductor lumped circuit model via ladder network. Circuit diagram of the electrical impedance of the semi-inductor model is defined by the ladder network resistance factor R and shunt inductance factor L (Weece and Allen, 2010).

Warren and LoPresti (2006) demonstrated that the Bessel function ratio in Vanderkooy's model (1989) can be expanded as a continued fraction expansion, as a diffusion ladder network, so that the electrical impedance can be represented by the circuit shown in Fig. 5. 
The semi-inductor model includes two parameters: the diffusion resistance $R$, and the shunt diffusive inductance $L$ which can be represented by the physical characteristics of the transducer. From Warren and LoPresti (2006) and Vanderkooy (1989), $R$ and $L$ are given by

$$
R=\frac{4 \pi n^{2} l}{\sigma}, L=\mu l n^{2} \pi r_{0}^{2},
$$

where $n$ is the number of coil windings, $l$ is the coil length, $\sigma$ is the conductivity of the pole structure, $\mu$ is the permeability of the pole structure, and $r_{0}$ the coil radius.

Although the combination of the resistor and the inductor should extend to infinity (more resistor-inductor pairs), these can only affect higher frequencies (a sufficient low frequency approximation). As shown in Fig. 5, Weece and Allen (2010) determined only 5 elements ( $\mathrm{L}, 2 \mathrm{R}, \mathrm{L} / 3,4 \mathrm{R}$, and L/5), and compared and denoted the network as a demagnetized condition of their a bone driver transducer.

\section{Methods}

Three different experiments were conducted in this study. First, we calculate the Hunt parameters of a BAR from electrical input impedance measurements. The calculation of Hunt parameters may be considered as a two-port Thevenin calibration of the receiver, since $Z_{e}, T$, and $Z_{a}$ characterize the initial electrical, acoustical and transitional properties of the unloaded receiver. Second, we measure of the receiver's diaphragm velocity in vacuum using a laser. This procedure was needed to verify the model, both the mechanical and electrical parts. The last step is the pressure measurement of the receiver using an ER7C probe microphone, (Etymotic Research). The resulting Thevenin pressure of the receiver from our transducer model and Hunt parameters is compared with this experimental pressure data. The detail of this result is discussed in Section 6 (model verification).

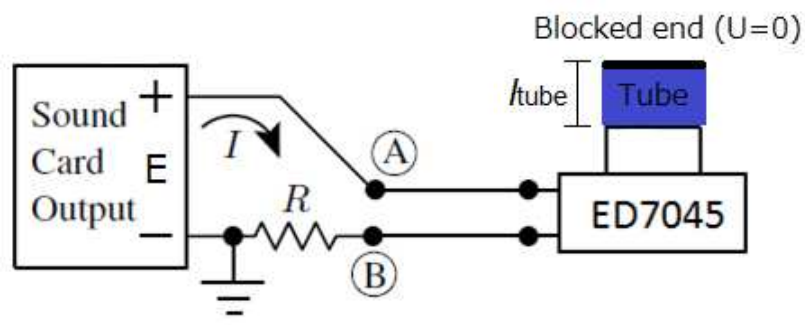

Figure 6: Experimental setup for the electrical input impedance measurement. $l_{\text {tube }}, \mathrm{E}, \mathrm{I}$, and $\mathrm{R}$ stand for the length of the tube, the voltage, the current and the reference resistance, respectively. We varied the experimental condition via changing $l_{\text {tube }}$ and, measured the voltage at two points (A, B) denoted as $E_{A}$ and $E_{B}$

\subsection{Electrical input impedance measurements for the Hunt parameter calculation}

Step 1 of calculating Hunt's parameters of the receiver requires a system for measuring electrical impedance as a function of frequency. As shown in Fig. 6, all stimulus signals were generated using a laptop sound card so that voltages could be measured and recorded. The stimulus waveform was a 24-bit, 2048-point frequency-swept chirp with a sampling rate of $48[\mathrm{kHz}]$. The signal-to-noise ratio was improved by looping the chirp and averaging between 10 and 100 consecutive frames, depending on the required SNR. The $\leqq 1$ volt chirp signal from an Indigo sound card (Echo Audio) was sent to the receiver which was in series with a known reference resistor $R(1 \mathrm{k}[\Omega]$, Fig. 6). The resistor was located between one of the receiver's terminals and the sound source ground. The measured electrical input impedance is expressed as:

$$
Z_{\text {in }}=\frac{E_{A}-E_{B}}{I}=\frac{E_{A}-E_{B}}{E_{B} / R}=R\left(\frac{E_{A}}{E_{B}}-1\right) .
$$

As shown in Fig. 7, eight different acoustic loads were attached to the end of the receiver output and eight corresponding electrical input impedances were recorded. Six of the seven tubes (excluding the longest length $6.11[\mathrm{~cm}]$ ) were used in the experiments: $0.25,0.37,0.88,1.24,2.39$ and $3.06[\mathrm{~cm}]$. The inner diameter of the tubes was about 

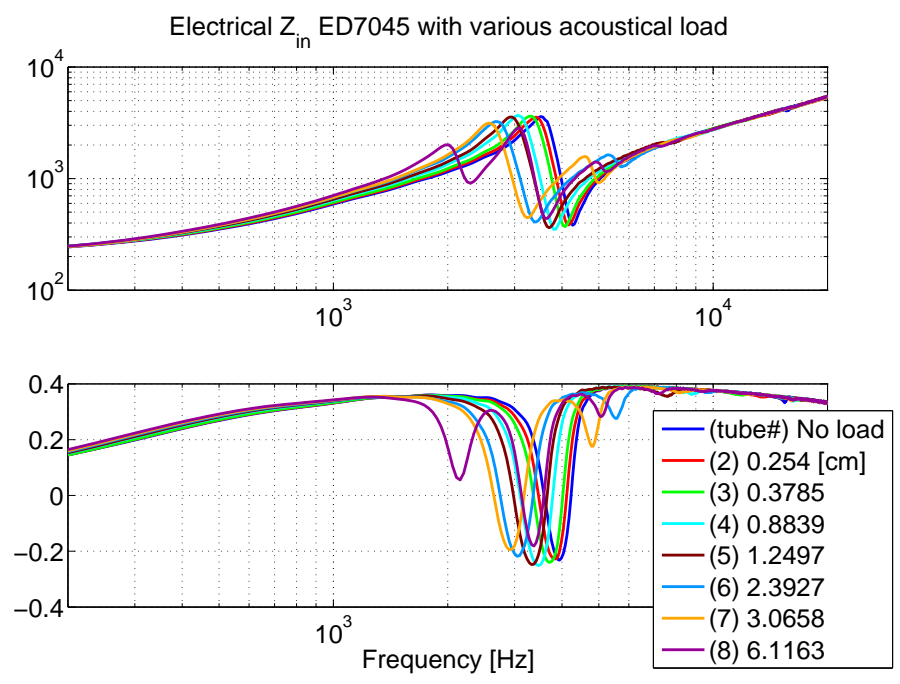

Figure 7: Measured $Z_{\text {in }}$ of ED7045 with the eight acoustical load conditions, blocked cavities. Different lengths of the tubes are used to vary the acoustical load. Three different known electrical input impedances are selected to calculate Hunt's parameters.

$\approx 1.5[\mathrm{~mm}]$, which is similar to the outer diameter of the ED receiver port. As three different measurements were required to calculate the three unknown Hunt parameters $\left(Z_{e}, Z_{a}, T_{a}\right)$ (Weece and Allen, 2010), three out of six tubes with different lengths were selected, resulting in $6 C 3=20$ possible combinations of the Hunt parameters. The results from every possible combination is not discussed in this paper; rather, we focus on the four calculated sets of Hunt parameters depicted in Fig. 11, shown as four curves in each of the six panels. The legend indicates the set of tubes corresponding to a given curve. The four curves are selected based on the consistency of the estimated Hunt parameters in their frequency response agreement up to $6[\mathrm{kHz}]$. When we calculated impedance of the testing tubes using a lossy tube model, we still see significant calibration error above $6[\mathrm{kHz}]$. The length of our testing tubes $\left(l_{t}\right)$ are short, and every experiment set-up was done manually, therefore measuring, attaching, and blocking the testing tubes by hands may contribute to this error. We categorized our testing tube lengths into short, medium and long tubes, and picked one of each to make a set of three tubes. An open circuit condition $(U=0)$ is applied, as the ends of the lossy tubes were blocked during the experiment. The characteristics of the resulting derived Hunt parameters are discussed in Section 4.

When the acoustic load impedance is unblocked, a small second resonance (SR) appears around 7.6[kHz], following the first resonance (FR) at $2.5[\mathrm{kHz}]$, as shown in Fig. 8 (a) (green). In fact, SR appears in every case in the figure, as clearly seen in the polar plot, Fig. 8 (b). The SR of the blocked case (red) is not noticeable in magnitude plot, but one sees the SR location from the phase, and the polar data. Note that a 'loop' in the polar data corresponds to the SR in the magnitude data. The vacuum data (blue) shows the biggest FR in magnitude (the largest circle in the polar plot), and the FR locates at the lowest frequency among all the other possible resonances.

Compared to the unblocked case (red), the SR frequency of the other two cases (blocked and vacuum) is above the frequency range of our interests. In detail, it has almost an octave difference $\left(\mathrm{SR}_{\text {unblocked }} \approx 7.6[\mathrm{kHz}], \mathrm{SR}_{\text {vacuum }} \approx\right.$ $\left.13.3[\mathrm{kHz}], \mathrm{SR}_{\text {blocked }} \approx 15.7[\mathrm{kHz}]\right)$. In addition, the size of $\mathrm{SR}_{\text {blocked }}$ is insignificant. For these reasons, we have ignored the SR effect when designing the BAR model.

\subsection{Laser vacuum measurements}

Figure 9 describes the experimental setup of the laser mechanical velocity measurement in the vacuum environment. In preparation for the laser measurement, the transducer's cover was carefully removed using a dental drill, to expose the diaphragm. A thin plastic window was glued on to reseal the case. The laser beam is finely focused on the diaphragm through the thin plastic window of the receiver and the measurement was made where the driver rod (Fig. 2) connects to the diaphragm. For the vacuum condition, air inside the receiver was evacuated prior to measurement. The ambient pressure was maintained at 0.003 [atm] during these measurements. The custom built vacuum system was used with a 'Sergeant Welch' vacuum pump and a 10-inch bell-shaped jar. A 'Polytec OFV-5000 Vibrometer controller' was used with a 10x-lens on the laser. The calibration factor for the laser velocity was 125 [mm/sec/volt]. 

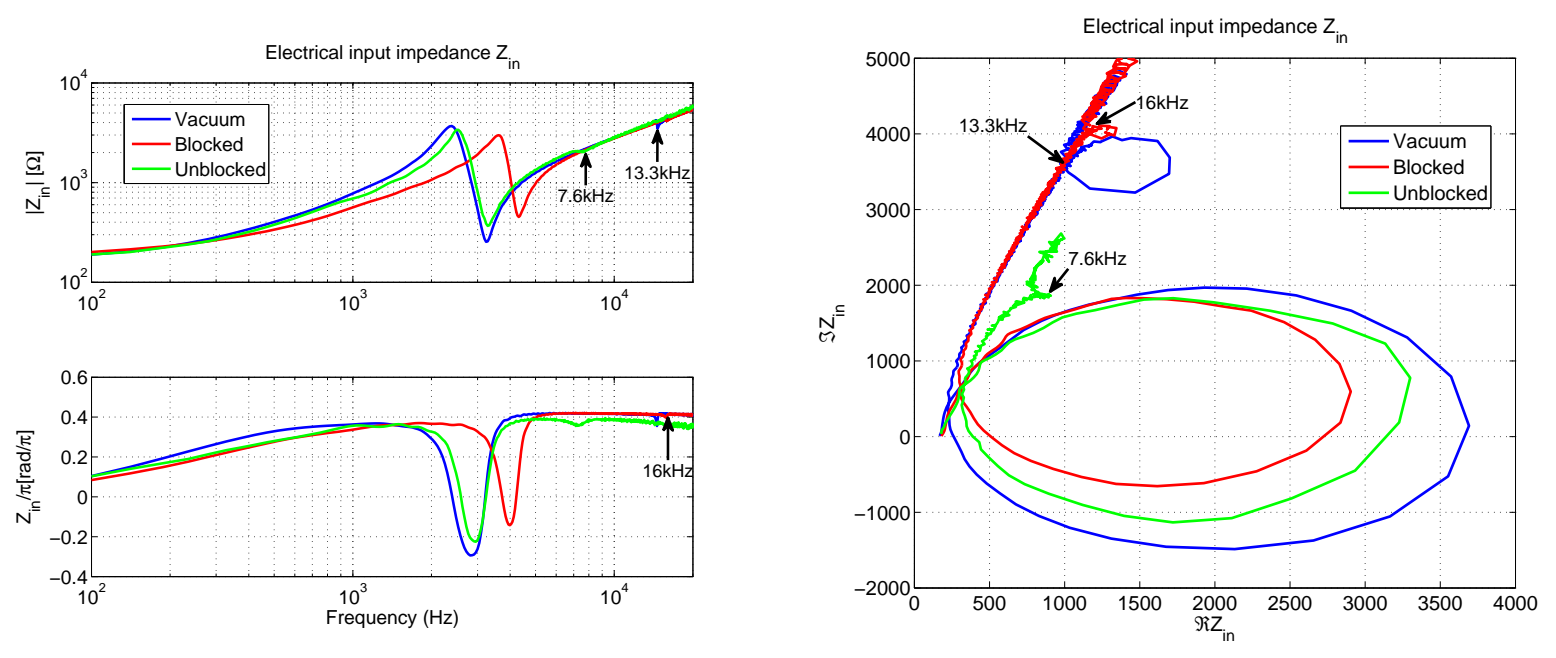

(a) Magnitude and phase of $Z_{\text {in }}$ of the ED7045 receiver

(b) $Z_{\text {in }}$ polar plot

Figure 8: This plot shows the electrical input impedance of the ED7045 receiver in blocked/unblocked port, and in-vacuum conditions. In the unblocked receiver port case, the FR moves to lower frequency $(2.5[\mathrm{kHz}])$ compared to the blocked case. The FR of vacuum stays at the lowest frequency, $2.3[\mathrm{kHz}]$. The frequency locations of SR for each curve are pointed by arrows in the figures. (a) Magnitude and phase of the electrical input impedance, (b) Polar plot of the electrical input impedance ( $\mathfrak{R} Z_{\text {in }}$ vs $\left.\mathfrak{J} Z_{\text {in }}\right)$.

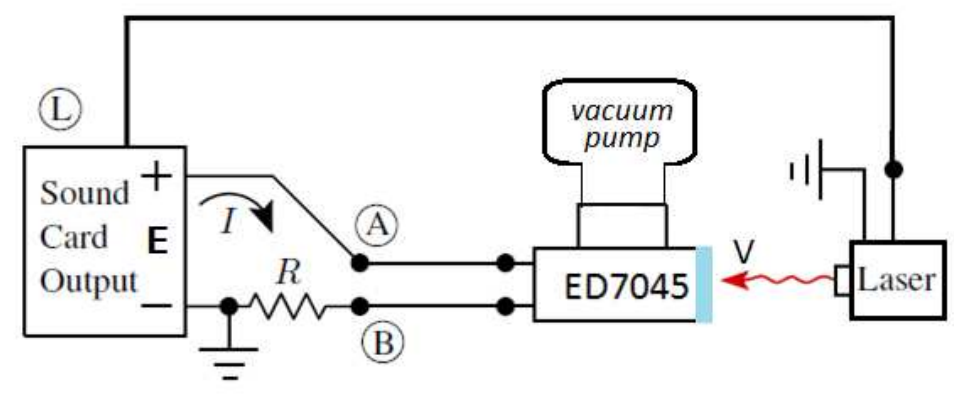

Figure 9: Experiment setup for the laser mechanical velocity measurement in vacuum. The circled 'L' means an input from the laser system. The laser beam is focusing on the plastic window of the transducer to measure the diaphragm velocity (V). 


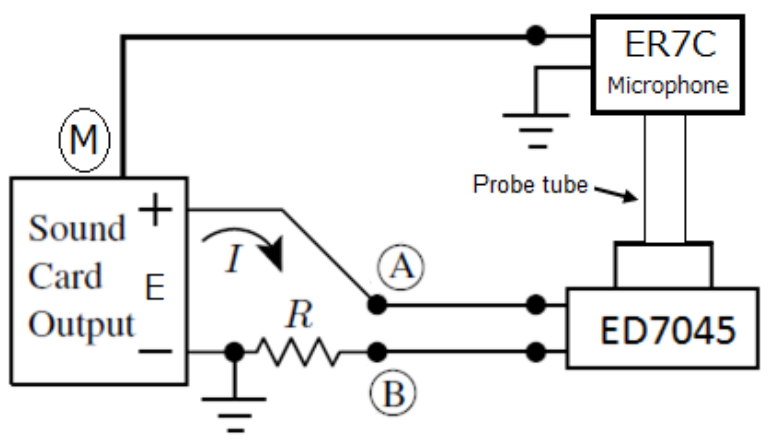

Figure 10: Experiment setup for pressure measurement. The circled ' $M$ ' means an input from the ER7C microphone.

The purpose of experiment three is to compare the output pressure to the model for $U=0$. An ER-7C probe microphone (Etymotic Research) was used for the transducer pressure measurement. The probe tube was .95 [mm] OD x .58 [mm] ID x $76[\mathrm{~mm}]$, and made of medical grade silicon rubber. In fact, it is impossible to connect a microphone probe with a perfectly blocked receiver $(U=0)$ due to the finite load impedance of the microphone. The space between the microphone's tube and the port of the receiver is minimized, and the tube and the port do not touch each other. The characteristic impedance of a tube $\left(Z c_{t u b e}\right)$ without loss is given by

$$
Z c_{\text {tube }}=\frac{\rho c}{\text { Area }_{\text {tube }}},
$$

where $\rho$ is the air density and $\mathrm{c}$ is the speed of sound $\left(1.2\left[\mathrm{~kg} / \mathrm{m}^{3}\right]\right.$ and $342[\mathrm{~m} / \mathrm{s}]$ at $20 C^{0}$, respectively). The diameter $(d)$ of the receiver's port and the microphone's tube are $d_{\text {receiver }}=1.4[\mathrm{~mm}]$ and $d_{m i c}=0.58[\mathrm{~mm}]$, thus the area of the receiver's port is about 6 times larger than the microphone's. Adding more consideration of the length of both cases, $Z c_{\text {mic_tube }}$ is much greater than $Z c_{\text {recever_port }}$. This shows that $Z c_{\text {mic_tube }}$ has a small loading effect on the source impedance of the receiver. Recognizing these experimental limitations prior to compare the measurement data to theoretical results will give us better understanding of the Thevenin pressure of the system.

In section 6.4, the calculated Thevenin pressure (per voltage denoted as $\mathrm{P} / \mathrm{E}$ ) is compared with the experimental pressure measurement.

\section{Hunt parameters $Z_{e}, Z_{a}$, and $T_{a}$}

\subsection{Calculation}

$Z_{e}, Z_{a}$ and $T_{a}$ are to be the estimated from $Z_{i n}$ given three different acoustic load conditions. Similar to Eq. 1, the BAR can be represented by its acoustic impedance matrix, in terms of the volume velocity $U$ into the port and the pressure $P$, as

$$
\left[\begin{array}{c}
E(\omega) \\
P(\omega)
\end{array}\right]=\left[\begin{array}{cc}
Z_{e}(s) & -T_{a}(s) \\
T_{a}(s) & Z_{a}(s)
\end{array}\right]\left[\begin{array}{c}
I(\omega) \\
U(\omega)
\end{array}\right] .
$$

The acoustic load impedance $Z_{L}$ is defined by Ohm's law as

$$
P \equiv-U Z_{L} .
$$

Combining Eq. 19 and Eq. 20 and solving for $U$ gives

$$
U=\frac{-T_{a} I}{Z_{L}+Z_{a}} .
$$


Replacing $U$ in Eq. 19 gives an expression for the loaded electrical input impedance $(U \neq 0)$

$$
Z_{\text {in }} \equiv \frac{E}{I}=Z_{e}+\underbrace{\frac{T_{a}^{2}}{Z_{L}+Z_{a}}}_{Z_{\text {mot }}},
$$

where $Z_{\text {mot }}$ is denoted as the motional impedance due to the acoustic load shown in the electric terminals (Hunt, 1954). Thus the $Z_{\text {in }}$ obtained through measurements depends on the acoustic load. Varying the acoustic load, which can be done by varying the length of the tube, gives different $Z_{\text {in }}$ value (Fig. 7). Since there are three unknown Hunt parameters, three different load conditions are required as discussed in the Appendix A.
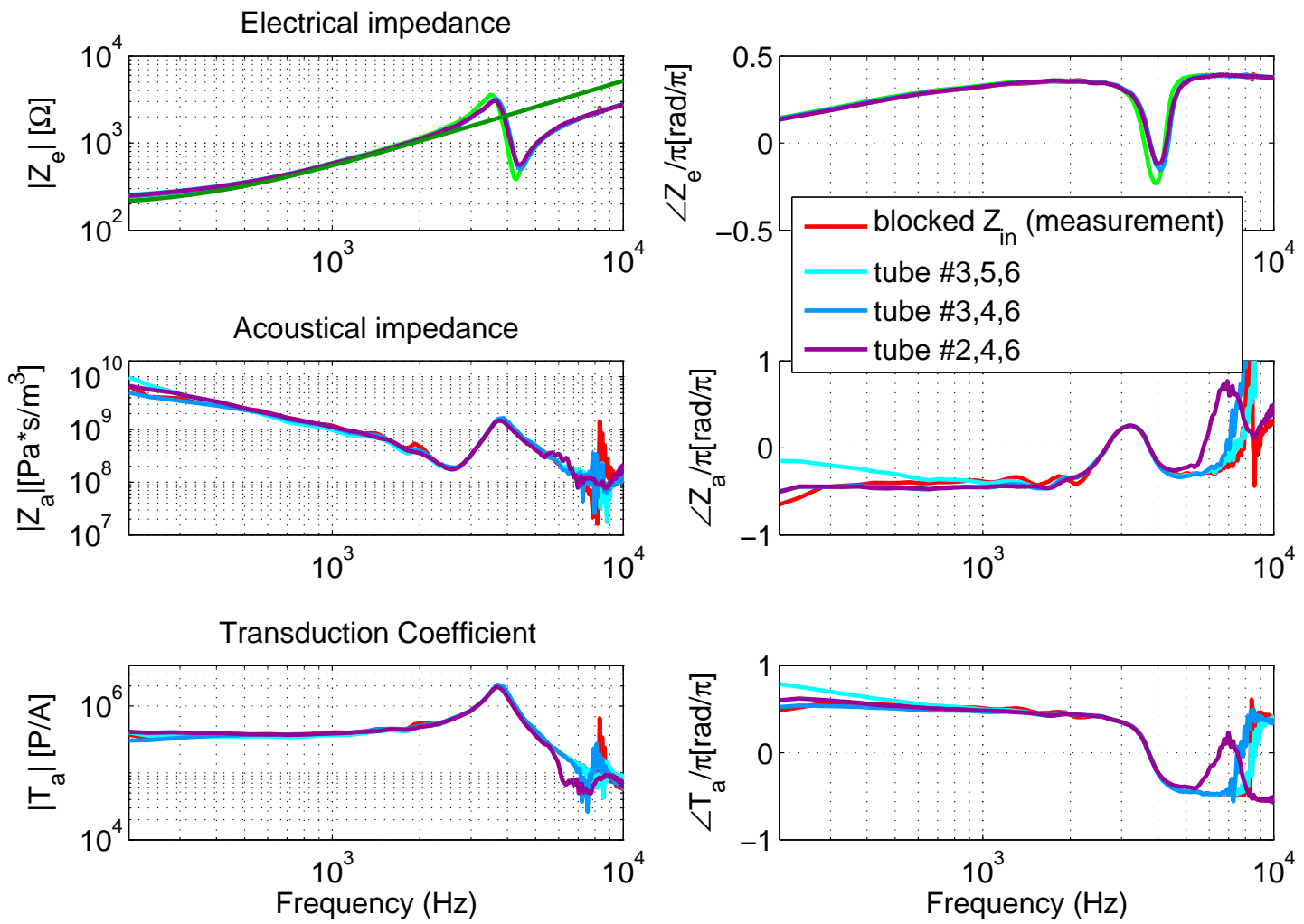

Figure 11: Calculated Hunt parameters $\left(Z_{e}, Z_{a}\right.$, and $\left.T_{a}\right)$ of the ED7045. Three conditions of $Z_{\text {in }}$ are required to find one set of the three Hunt parameters. The choice of $Z_{i n}$ for each calculation is shown in the legend, as tube numbers. The specific length of each numbered tube is described in Fig. 7. $Z_{\text {in }}$ which is measured by blocking the receiver's port $(U=0)$ is plotted with $Z_{e}$. Note that $Z_{i n} \rightarrow Z_{e}$ as $U \rightarrow 0$. While the experimental (blocked $Z_{i n}$ ) and calculated $\left(Z_{e}\right)$ values are not exactly the same, they are similar. For both cases, $Z_{m o t}=0$, (when $\left.U=0\right)$ since $Z_{L}=0$.

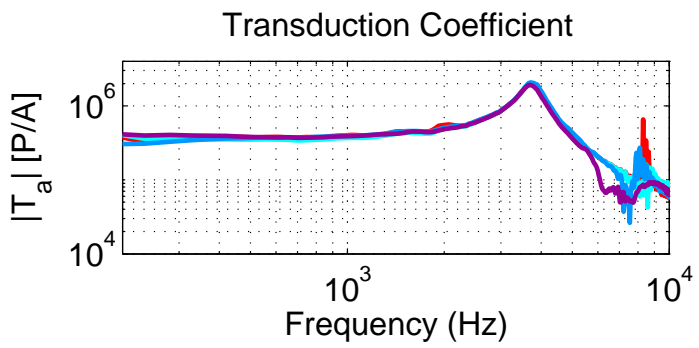

\subsection{Investigations}

The calculated Hunt parameters of the BAR derived from various $Z_{i n}$ in Fig. 7 are shown in Fig. 11. Some considerations for the Hunt parameters of the BAR are as follows:

1. $\mathbf{Z}_{\mathrm{e}}$ : Compared to $Z_{a}(s)$ and $T_{a}(s), Z_{e}(s)$ has the smallest dependency on the choice of load cavities (the three of six chosen load impedances: loads (2)-(7) in Fig. 7). Below 200[Hz], $Z_{e}(s)$ converges to a fixed resistance (ED7045: $\approx 195[\Omega]$ ). The frequency range between $0.5-2.5[\mathrm{kHz}]$ is proportion to ' $\mathrm{s}$ ' $\left(Z_{e}\right.$ shows a constant slope in this frequency range). It is not clearly shown at frequencies below $10[\mathrm{kHz}]$, however when the frequency increases 
more, the slope of $Z_{e}$ approaches that of ' $\sqrt{s}$ '. Better evidence of ' $\sqrt{s}$ ' domination at high frequency is shown in section 6.2 in the polar plot. These frequency dependant impedance behavior (proportional to a constant, ' $\mathrm{s}$ ' and ' $\sqrt{s}$ ') is determined by the coil properties, which are closely related the DC resistance inductance and the semi-inductance.

2. $\mathbf{Z}_{\mathrm{a}}$ : For the frequencies below $2.5[\mathrm{kHz}], Z_{a}$ is stiffness dominated (i.e., a capacitance), and between $2.5-4[\mathrm{kHz}]$ is dominated by the mass of the diaphragm and armature. Those properties determine the first anti-resonance (zero, near $2.5[\mathrm{kHz}]$ ). The resonance (pole) at $3.7[\mathrm{kHz}]$ is the frequency where the transfer impedance, $T_{a}$, is maximum. The pole of $Z_{e}$ is also introduced in this frequency point, however $T_{a}$ and $Z_{a}$ are tied more closely, they move together when the set of Hunt parameter is changed while $Z_{e}$ is almost identical over every set of the Hunt parameters (Fig. 13). Above $4[\mathrm{kHz}]$ the transmission line and acoustic properties dominates given the small volume inside the receiver. Error above $6-7[\mathrm{kHz}]$ is primarily caused by the experimental limitations, such as manual manipulation of the tubes.

3. $\mathbf{T}_{\mathbf{a}}$ : It is nearly constant below $2-3[\mathrm{kHz}]$ and is $4 \times 10^{5}[\mathrm{~Pa} / \mathrm{A}]$ at $1[\mathrm{kHz}]$. The phase shift in $T_{a}$ is due to acoustic delay. Although the frequencies above $6[\mathrm{kHz}]$ are obscured by the noise, $T_{a}$ seems to behave as an all-pole function, which depend on the system delay $\tau$. To account for this delay, a transmission line (Tx line) is added to the acoustic model, as shown in Fig. 12.

\section{Results: Proposed receiver model}

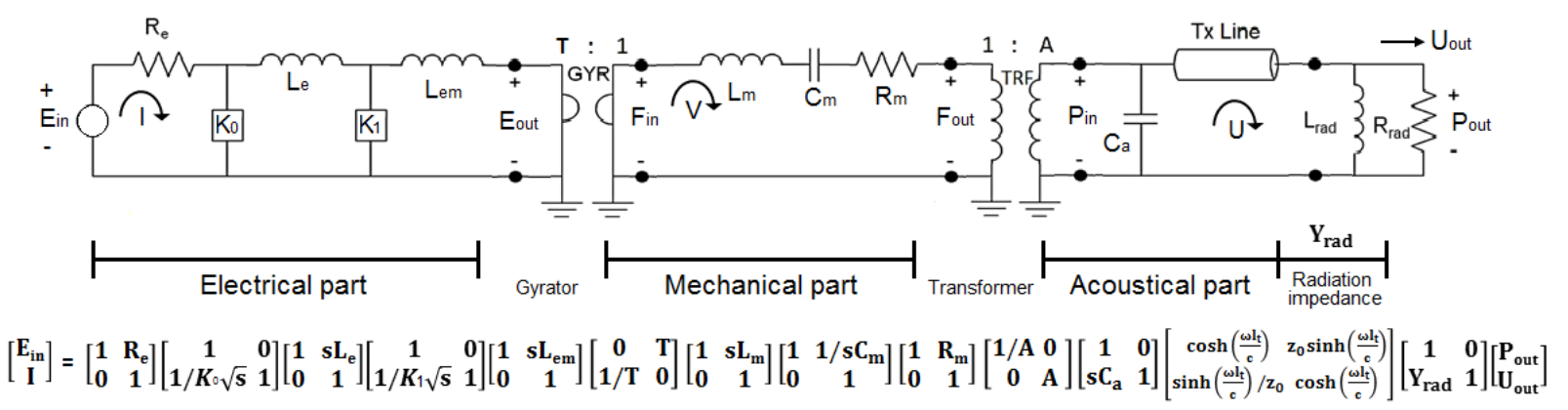

Figure 12: Final circuit model of the BAR, along with the equivalent ABCD matrix representation. The electrical and the mechanical circuits are coupled by a gyrator (GYR), while a transformer (TRF) is used for the coupling of the mechanical and the acoustical circuits. The input and output potentials for each section (E: voltage, F: force, P: pressure) are specified in the model. I (current), V (particle velocity), and U (volume velocity) represent the flow of each part. By computing the defined ABCD matrix and converting the result to the impedance matrix via Eq. 2, Hunt parameters of the transducer model may be calculated.

Figure 12 shows the final transducer ABCD model. The electrical circuit elements are shown to the left of the gyrator. $R_{e}$ and $L_{e}$ are approximated to the DC resistance and the self-inductance of the wire inside of the transducer, respectively. Current through the coil generates the magnetic flux $\left(\Phi=\bar{B} S_{a}\right.$, Eq. 7). The magnetic field diffusion (eddy-current) on the armature (a conductor) causes the first eddy current denoted as $K_{0}$ in Fig. 12. On account of the imperfect electro-magnetic linking between the coil and the armature (primary caused by the gap), the leakage inductance $L_{e m}$ is represented as an additional inductive component along with the primary inductance, $L_{e}$. This leakage inductance results in a second eddy-current leading to $K_{1} \sqrt{s}$. The shunt semi-inductor $\left(K_{0}\right)$ in series with the DC resistor $R_{e}$ results in ' $Z_{e} R_{e}$ ' $\propto s$ at low frequencies $(<1[\mathrm{kHz}])$ and $\propto \sqrt{s}$ at high frequencies.

There is a transition frequency $\left(f_{t}=\frac{1}{2 \pi}\left(\frac{K}{L}\right)^{2}\right)$ between the normal inductor $(\mathrm{L})$ and the semi-inductor $(\mathrm{K})$. Since we used two of the each inductor and the semi-inductor (total 4) for the armature receiver model, it is tricky to calculated the exact $f_{t}$ from these four components. As we discussed in section 4.2, it is hard to draw a fine line of the transition between ' $\mathrm{s}$ ' and ' $\sqrt{s}$ ' on Fig. 11 within the provided frequency range $(100-10[\mathrm{kHz}])$. However as shown in Fig. 14 polar plot, the slope of the impedance is approaching $\sqrt{s}$ as $\omega$ increases. Based on Thorborg et al. (2007), the $f_{t}$ of a dynamic loudspeaker is $100-200[\mathrm{~Hz}]$, which means the $f_{t}$ for the balanced armature receiver is much higher than for 


\section{Electrical elements}

$$
\begin{aligned}
& R_{e}=190[\Omega], \\
& L_{e}=0.05[\mathrm{H}], K_{0}=32 \text { [Semi-Henry], } \\
& L_{e m}=0.011, K_{1}=200 \text { [Semi-Henry] } \\
& \text { GYR }=7.5
\end{aligned}
$$

\section{Mechanical elements}

$$
\begin{aligned}
& C_{m}=1.2 \mathrm{e}-3[\mathrm{~F}], \quad L_{m}=4.4 \mathrm{e}-6[\mathrm{H}], R_{m}=0.003[\Omega] \\
& \text { TRF }\left(\mathbf{1} / \text { Area) }=1 /\left(2.9 e^{-6}\right)\right.
\end{aligned}
$$

\section{Acoustical elements}

$$
\begin{aligned}
& C_{a}=6.2 \mathrm{e}-15[\mathrm{~F}] \\
& \text { Tx Line: } \omega / c, z_{0}=6 \mathrm{e} 8[\mathrm{~kg} / \mathrm{sec}], l_{t}=1 \mathrm{e}-4[\mathrm{~m}]
\end{aligned}
$$

Table 1: Specific parameters that are used for the suggested model (Knowles BAR ED7045). c is the speed of sound in the air (334.8 [m/s]), $\omega / \mathrm{c}$, $z_{0}$, and $l_{t}$ are the propagation function, specific characteristic impedance and length of the transmission line, respectively. GYR and TRF stand for the gyrator and the transformer. All model parameters were found by minimizing the RMS error between the model and electrical input impedance measurements of the receiver.

the loudspeaker.

$$
\left.Z_{e}(s)\right|_{T=0 \text { or } F_{i n}=0}=R_{e}+\frac{K_{0} L_{e} L_{e m} s \sqrt{s}+\left(K_{0} K_{1} L_{e}+K_{0} K_{1} L_{e m}\right) s}{L_{e} L_{e m} s+\left(K_{1} L_{e m}+K_{1} L_{e}+K_{0} L_{e m}\right) \sqrt{s}+K_{0} K_{1}} \propto\left\{\begin{array}{ll}
R_{e}+\frac{1}{K_{0} K_{1}} & \omega \rightarrow 0 \\
R_{e}+K_{0} \sqrt{s} & \omega \rightarrow \infty
\end{array} .\right.
$$

The gyrator relates the electrical and the mechanical sections with parameter $T$. All other parameters in Eq. 13 are measurable (at least estimable) values, except for $F_{M}$. The number of coil turns ' $n$ ' is related to the length of the wire $l$ and the coil diameter $\left(d_{c o i l}\right)$. For instance, the wire inside the ED7045 receiver is made of 49 AWG copper, which has a resistivity of $26.5[\mathrm{Ohm} / \mathrm{m}]$. Since the measured DC resistance of the receiver is around $195[\Omega]$ we can estimate that the length of the wire is approximately $7.35[\mathrm{~m}]$. If we know the diameter of the winding coil, the ' $\mathrm{n}$ ' can be approximately calculated by dividing ' $l$ ' by the circumference of the coil. An inductance of a motor is related to the number of turns ' $n$ ' via,

$$
L_{\text {motor }}=\left(L_{e}+L_{e m}\right) \propto n^{2} .
$$

In general, the $d_{\text {coil }}$ is much greater in the case of the moving coil speaker while ' $l$ ' is longer in the BAR. Therefore we can expect a larger ' $\mathrm{T}$ ' value for the BAR $\left(n \propto l, 1 / d_{\text {coil }}\right)$. Because of the separation between the coil and the diaphragm in the BAR, a larger ' $n$ ' or a mass of the coil has nothing to do with the movement of the armature, as the coil is not dynamic (in the case of a loudspeaker, the coil moves up-and-down, surrounding a pole piece, thus adding mass).

To the right of the gyrator are the mechanical and acoustical sections of the transducer. We can simply describe the mechanical section as composed of a series combination of the armature and the diaphragm's stiffness, mass and damping. The transformer's coupling ratio of the acoustic side to the mechanical side is related to the diaphragm's area. The capacitor $\left(C_{a}\right)$ and a transmission line in the acoustical part account for the small volume and sound delay in between the port and the diaphragm. Because we are using a gyrator, it is not necessary to use the mobility analogy method (Hunt, 1954).

The Thevenin pressure of the BAR is defined when the volume velocity $(U)$ at the port is zero ('blocked' port), meaning the load impedance is set to zero.

\section{Verification of the transducer design}

Several comparisons are made to verify that the transducer model (Fig. 11, 12. First, Hunt parameters are calculated from the model to support the transfer relation between electrical and acoustical parts (Section 3.1) as outlined 
in Appendix A. The mechanical part of the transducer model was verified by conducting laser mechanical velocity measurements in a vacuum condition (Section 3.2). Along with these results, we simulated the Thevenin pressure of the transducer from our model and compared the result to the pressure measurement (when $U=0$ ) (Section 3.3). These three comparisons (electrical, mechanical, and acoustical) verify the transducer model suggested in Fig. 12.

\subsection{Hunt's parameters comparison}

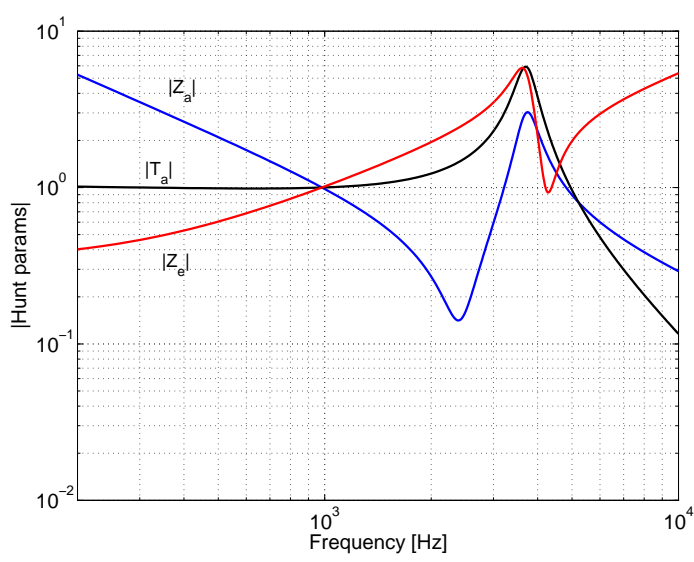

(a) Hunt parameters from the model

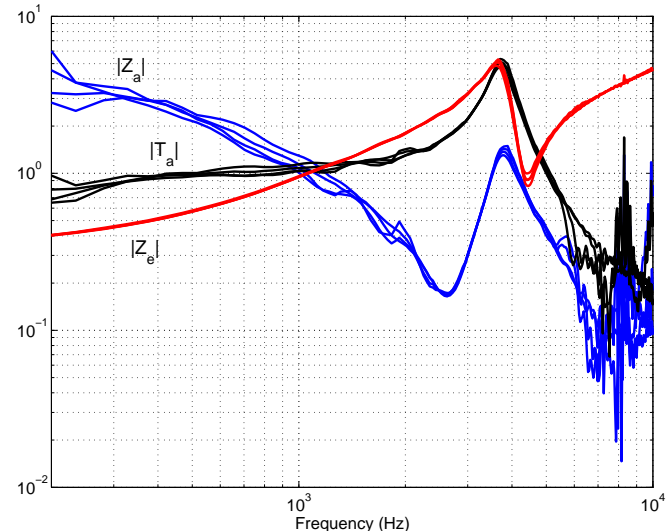

(b) Hunt parameters from measurement

Figure 13: Comparison of Hunt parameters $\left(Z_{e}\right.$ (red), $T_{a}$ (black), and $Z_{a}$ (blue)) from the model (a) and the measurements (b). Any significant differences between the model and the data occur above $6[\mathrm{kHz}]$. All parameters are normalized to their $1[\mathrm{kHz}]$ values.

The Hunt parameters, from the model and the experimentally calculation, are compared to each other in Fig. 13. The discrepancies of $Z_{a}$ above $6-7[\mathrm{kHz}]$ are presumably caused by the manual adjustment of the experimental conditions. This error is insignificant in $Z_{e}$. However the small noise in electrical impedance amplifies as the parameter is far from the electrical side. In other words, we can see the largest noise in acoustical parameter $\left(Z_{a}\right)$, as the transition order goes from $Z_{e} \rightarrow T_{a} \rightarrow Z_{a}$.

Another interesting observation is the resonant frequency of the three parameters. The frequency of the pole $\left(f_{p}\right)$ for all parameters looks almost identical, especially $Z_{e} . Z_{a}$ and $T_{a}$ slightly differ by the set, but the $f_{p}$ of the two parameters occurs at the same location in the same set of Hunt parameters. The three parameters assume the zero-loaded condition which means, in theory, the $f_{p}$ should be identical for all cases. Because of measurement differences, this is not exactly the case. This resonant location can be interpreted as one of the important characteristic of an electro-magnetic transducer.

\subsection{Electrical impedance in vacuo}

The vacuum condition only includes mechanical and electrical circuits. The acoustical part in the transducer model is removed for the vacuum simulation, while all the electrical and mechanical parameters in Fig. 12 during the experiments remain the same as the no-vacuum condition.

In Fig. 14, the simulated electrical input impedance results are expressed in two ways; the magnitude-phase and the real-imaginary parts. For both the vacuum and the blocked port condition, the model (solid lines) and the experiment result (dashed lines) show reasonable agreement.

The transducer model, including acoustical elements ('blocked' output port) is in red, and the model excluding acoustical elements (vacuum condition) is in blue. Both cases give similar shape, a pole, followed by a zero, with increasing frequency $(\approx 890[\mathrm{~Hz}]$ in vacuum, $\approx 750[\mathrm{~Hz}]$ in blocked case). We conclude that the trapped air (between the diaphragm and the port of the receiver) influences the resonance by pushing it to higher frequencies due to the increased stiffness to mass ratio. Also because of the acoustical properties (including mechanical-acoustical coupling), the magnitude of the vacuum resonance reduced by $1.9 \mathrm{~dB}$ compared to the blocking the receiver's output port (in the air). 

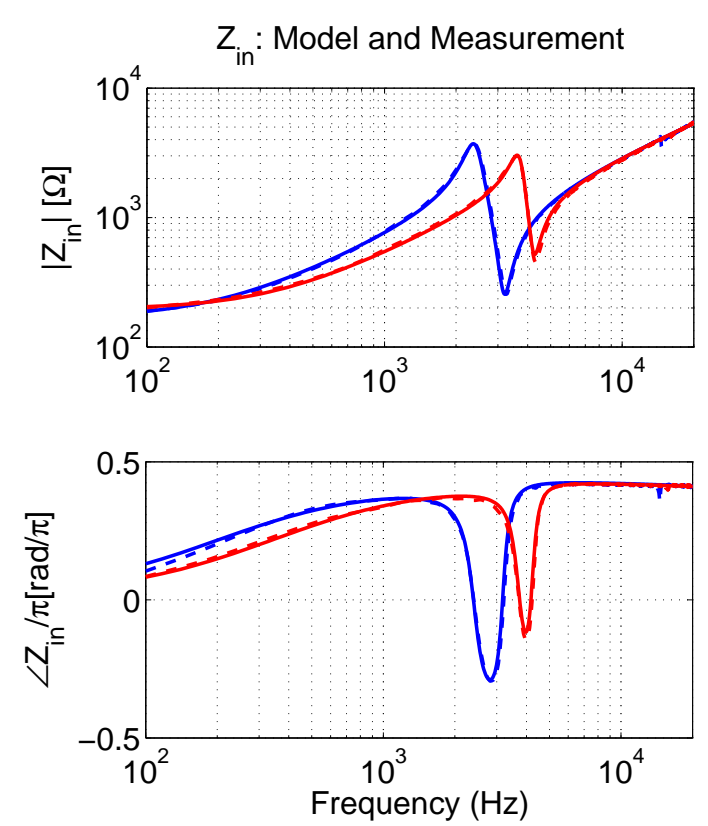

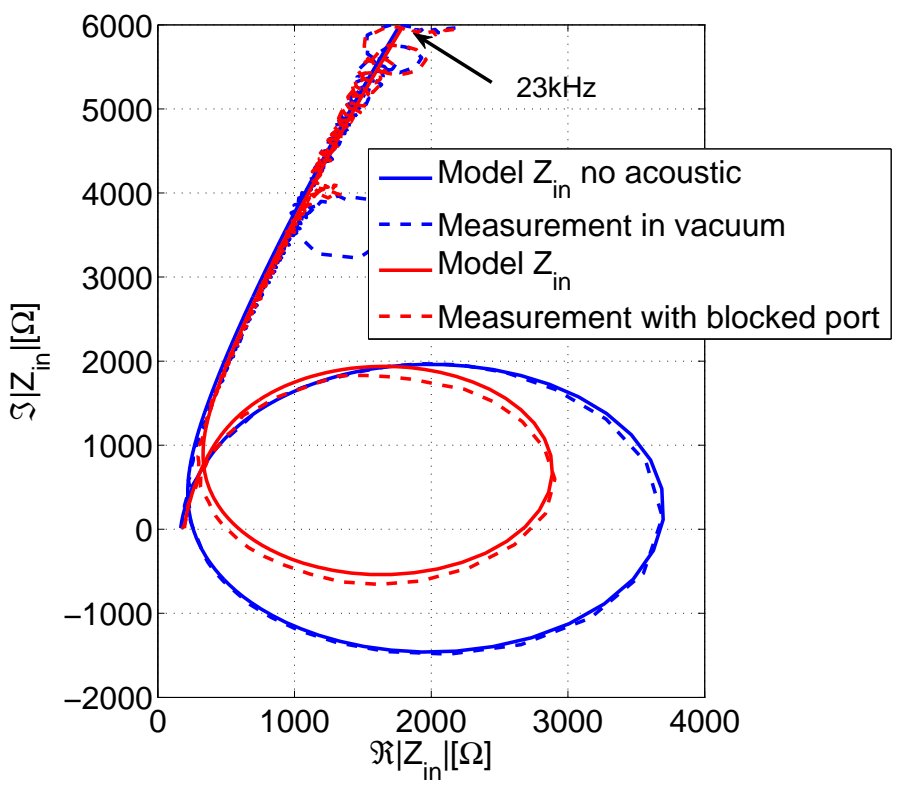

Figure 14: Comparison between suggested model and real electrical input impedance measurement of a balanced armature hearing-aid receiver (Knowles, ED7045). Blue and red colors represent vacuum and non-vacuum (with air) conditions respectively. And the dashed lines stand for the experimental result whereas the single lines show the model results. For the vacuum experiment, the actual pressure is 0.003 [atm]. The left panel shows the magnitude and the phase of each condition and the real and imaginary parts of the same data are plotted in the right panel. Up to 23[kHz], the experimental data is in good agreement with the modeling result (The sampling rate is $48[\mathrm{kHz}]$, therefore the maximum frequency is $24[\mathrm{kHz}]$ ). In the polar plot, when it goes to the higher frequencies, the impedance behaves as $\sqrt{s}$.

By looking at the polar plot (the right panel in Fig. 14, we can clearly see that the high frequency impedance is dominated by $\sqrt{s}$, the evidence of the eddy-current in the BAR. The small loops appeared in high frequencies in the measurement data are interpreted as artifacts of the experiment.

\subsection{Mechanical velocity measurement using Laser in vacuo}

As shown in Fig. 15, the mechanical velocity is also calculated from the transducer model and compared with the laser velocity measurement result. The model and the experiment are well matched below 10[kHz]. The large 20dB discrepancy above $10[\mathrm{kHz}]$ seems related to the SR observed in the electrical input impedance measurement in the vacuum condition (Fig. 14, the dashed blue line: a second loop appears at around $15[\mathrm{kHz}]$ in polar plot).

Other small magnitude difference is observed; the laser measured data has less damping on the FR and the low frequency area. There are some possible solutions to improve the model. First, when we make the laser measurement, we can focus on the laser beam in various places on the diaphragm and average out those data to see the overall velocity behavior of the diaphragm. It is true that we can get a slightly different velocity depending on the place where the laser focus is on. In our experiment as explained in method section, we put the laser's focus near at the rod (where the armature and the diaphragm is connected). And secondly, we could add or remove mechanical damping in the transducer model (i.e., increasing or decreasing the value of $R_{m}$ in our model Fig. 12) relative to the present value.

The mechanical velocity is calculated by assuming the force $(\mathrm{F})$ in vacuum is zero. In reality, it is impossible to reach an absolute vacuum condition. Our experiment condition of 0.003 [atm] seems adequate to understand the nature of the mechanical velocity of the transducer as the measurement sees reasonable agrement with the model.

\subsection{Thevenin pressure comparison}

The model pressure is plotted in Fig. 16 along with measured pressure data. Two indirect pressure estimation methods are used; one using the Hunt parameters, and the other using the simulation of our transducer model. There 


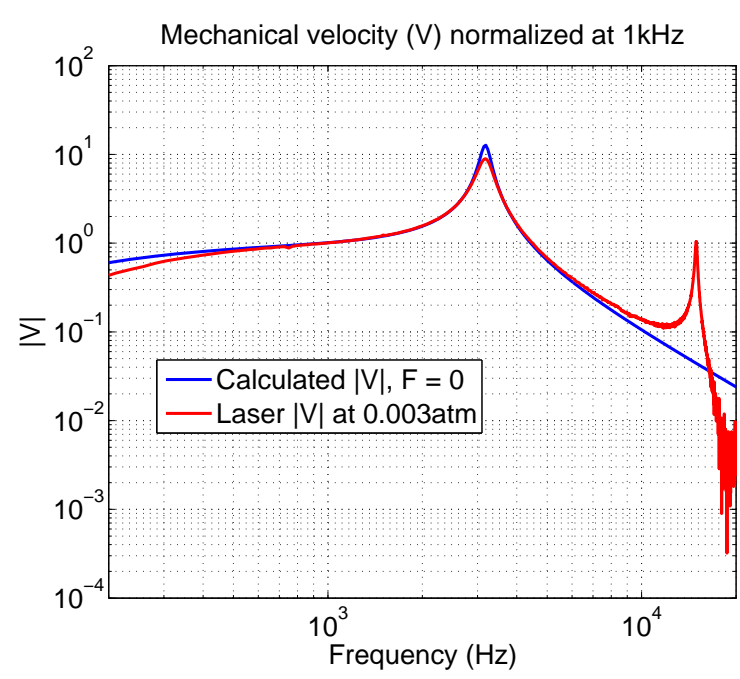

Figure 15: Comparison of the diaphragm (mechanical) velocity between the transducer model and the laser measurement in vacuum, the pressure
$\mathrm{P}$ is zero. For the model simulation, the acoustical part in Fig. 12 is not included. The laser measurement was performed after pumping out the air in the receiver. All values are normalized to one at $1[\mathrm{kHz}]$.

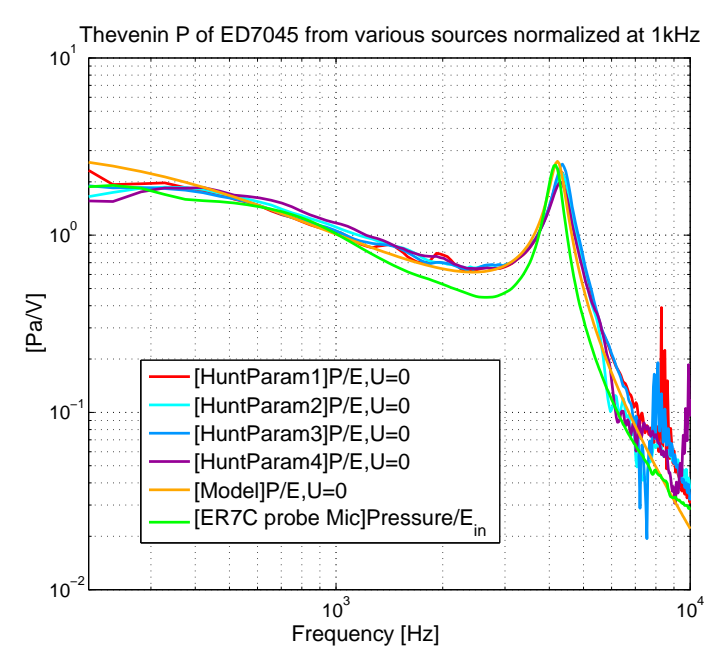

Figure 16: Comparison of Thevenin pressure (per voltage) data from various sources. There are 6 different lines, the first 4 lines are calculated from the electrical experiments (Hunt parameters), and the orange colored line is estimated from the model. The last pressure data (in light-green) are taken from the pressure measurement and are divided by the electrical input voltage of the receiver. All data assume the blocked condition, $\mathrm{U}=0$ (see text). Every value is normalized to one at $1[\mathrm{kHz}]$.

is a reasonable agreement among these measures up to $6-7[\mathrm{kHz}]$. The mathematical definitions of these data are the Thevenin pressure per unit voltage $(\mathrm{P} / \mathrm{E})$, with a zero volume velocity $(U=0)$,

$$
\frac{T_{a}\left(=\left.\frac{P}{I}\right|_{U=0}\right)}{Z_{e}\left(=\left.\frac{E}{I}\right|_{U=0}\right)}=\left.\frac{P}{E}\right|_{U=0} .
$$

Note that $\frac{P}{I}$ and $\frac{P}{E}$ differ in the theoretical meaning as well as in the definition; $\left.T_{a} \equiv \frac{p}{I}\right|_{U=0}$ is one of the Hunt parameter, while $\left.\frac{T_{a}}{Z_{e}} \equiv \frac{P}{E}\right|_{U=0}$ is more realistic experimental function, since one typically uses a voltage drive.

For the comparison, the pressure data is divided by the voltage $\left(E_{i n}\right)$ across the two electrical terminals of ED7075 (A and B in Fig.10) when $U=0$. The data from section 3.1 is imported for $E_{\text {in }}$ as we can not assume $U=0$ in this pressure measurement.

The green line in Fig. 16 shows the pressure data derived from the ER-7C probe microphone. Note that other than the direct pressure measurement (green), all responses are derived from the Hunt parameter calculation introduced in the Appendix, using the 'electrical input impedance measurements' for acoustical loads.

\section{Conclusion}

In this study, we have discussed the critical elements of a BAR including a gyrator, and a semi-inductor along with the two-port network properties. Starting by solving the Hunt parameters of the receiver, we have proposed a new circuit model which contains these elements, the gyrator and the semi-inductor. An intuitive design of an electromagnetic transducer has been enabled by using the gyrator. Moreover we could expect an elaborated high frequency matching by using the semi-inductors, especially the electrical impedance.

The model has been verified by comparing the experimental data (obtained from laser, vacuum, and pressure measurements) to theoretical data (obtained through model simulations). All the comparisons are in good agreement with the experimental results, especially the electrical input impedance data introduced in Fig. 14 matching up to $23[\mathrm{kHz}]$. The major advantages of the proposed receiver model is that the acoustic Thevenin pressure can be calculated solely from electrical input impedance measurements. 
Further study may improve the model for more elaborate match, such as the secondary loop as discussed in section 6.2. Also studying for a demagnetized transducer $(T=0)$, we could explore the nature of the electromagnetic transducer by looking at the $Z_{e}$ is dominated only by the electrical parameters. Analyzing the $f_{p}$ (introduced in section 6.1) from various transducers could be another interesting topic for the future study.

\section{Appendix A.}

Equation 22 includes three unknown Hunt parameters $\left(Z_{e}, Z_{a}\right.$ and $\left.T_{a}\right)$ that we wish to find. In order to solve for three unknown parameters, 3 different electrical input impedances $\left(Z_{i n \mid A}, Z_{i n \mid B}\right.$, and $\left.Z_{i n \mid C}\right)$ are measured corresponding to three known loads, A, B, and C. The load conditions differ in a length of the tubing, attached to the receiver's port. Each tube has different impedance denoted as $Z_{L \mid A}, Z_{L \mid B}$, and $Z_{L \mid C}$, where $Z_{L \mid A}=Z_{0} \operatorname{coth}(a \cdot$ tube_length) (for the blocked-end tube, $U=0), Z_{0}$ is the characteristic impedance of a tube, and ' $a$ ' is the complex propagation function. ' $a$ ' and $Z_{0}$ parameters assume viscous and thermal loss (Keefe, 1984). In 20 $C^{\circ}$ room temperature, $\mathrm{c}=334.8[\mathrm{~m} / \mathrm{s}]$.

Substituting these for $Z_{L}$ in Eq. 22:

$$
Z_{i n \mid A}=\frac{E}{I}=Z_{e}+\frac{T_{a}^{2}}{Z_{L \mid A}+Z_{a}}
$$

$$
Z_{i n \mid B}=\frac{E}{I}=Z_{e}+\frac{T_{a}^{2}}{Z_{L \mid B}+Z_{a}}
$$

$$
Z_{i n \mid C}=\frac{E}{I}=Z_{e}+\frac{T_{a}^{2}}{Z_{L \mid C}+Z_{a}} .
$$

Given these three measured impedances, we can solve for $Z_{a}, T_{a}$, and $Z_{e}$ via the following procedure:

1. Substract two electrical impedance measurements to eliminate $Z_{e}$, such as

$$
Z_{i n \mid C}-Z_{i n \mid A}=\frac{T_{a}^{2}}{Z_{a}+Z_{L \mid C}}-\frac{T_{a}^{2}}{Z_{a}+Z_{L \mid A}} .
$$

2. Take the ratio of various terms as defined by Eq. A.2,

$$
\left(\frac{Z_{a}-Z_{L \mid B}}{Z_{i n \mid C}-Z_{i n \mid A}}\right)=\left(\frac{Z_{i n \mid A}-Z_{i n \mid C}}{Z_{i n \mid B}-Z_{i n \mid C}}\right)\left(\frac{Z_{L \mid C}-Z_{L \mid B}}{Z_{L \mid C}-Z_{L \mid A}}\right) .
$$

From this we may find the first unknown $Z_{a}$,

$$
Z_{a}=\frac{\left(Z_{i n \mid A}-Z_{i n \mid C}\right)\left(Z_{L \mid C}-Z_{L \mid B}\right)\left(Z_{i n \mid C}-Z_{i n \mid A}\right)}{\left(Z_{i n \mid B}-Z_{i n \mid C}\right)\left(Z_{L \mid C}-Z_{L \mid A}\right)}+Z_{L \mid B} .
$$

3. Next we find $T_{a}$ by substituting $Z_{a}$ into Eq. A.2

$$
T_{a}=\sqrt{\frac{\left(Z_{i n \mid C}-Z_{i n \mid A}\right)\left(Z_{a}+Z_{L \mid C}\right)\left(Z_{a}+Z_{L \mid A}\right)}{Z_{L \mid A}-Z_{L \mid C}} .}
$$

4. Finally $Z_{e}$ is given by Eq. A.1

$$
Z_{e}=\left(\frac{T_{a}^{2}}{Z_{L \mid A}+Z_{a}}\right)-Z_{i n \mid A} .
$$




\section{Appendix B.}

If a system has a transmission line between its input and output (such as a vocal tract in human speech), the transfer function of the system can be represented as an all-pole system. This can be easily proven using the ABCD matrix relationship of a transmission line.

$$
\left[\begin{array}{c}
E_{1} \\
I_{1}
\end{array}\right]=\left[\begin{array}{cc}
\cosh \left(\frac{\omega l_{t}}{c}\right) & z_{0} \sinh \left(\frac{\omega l_{t}}{c}\right) \\
\sinh \left(\frac{\omega l_{t}}{c}\right) / z_{0} & \cosh \left(\frac{\omega l_{t}}{c}\right)
\end{array}\right]\left[\begin{array}{c}
E_{2} \\
-I_{2}
\end{array}\right],
$$

where ' $\underline{\omega}$ ' is the propagation function, 'c' is speed of sound, $l_{t}$ is length, $z_{0}$ is the characteristic impedance of the transmission line, $E_{1}, E_{2}, I_{1}$ and $I_{2}$ stand for the voltage and the current at input and output ports respectively. In open circuit condition, the transfer function $H=\left.\frac{E_{2}}{E_{1}}\right|_{I_{2}=0}$ is

$$
H=\frac{1}{\cosh \left(\omega l_{t} / c\right)}
$$

which is an all-pole system. Along with our modeling result, the transfer function simulation of the SPICE model in Fig. 4 also exhibits all-pole behavior. This all-pole property of the transduction coefficient ' $T_{a}$ ' has good agreement with results from Weece and Allen (2010).

\section{Acknowledgments}

The authors are grateful to HSR group, especially Hanul Kim along with Daniel Warren, Chris Jones and Tom Miller at Knowles Electronics (Itasca, IL) for their gracious help with the vacuum and laser measurements.

\section{References}

B. B. Bauer. A miniature microphone for transistor amplifiers. The Journal of the Acoustical Society of America, 25 (5):867-869, 1953.

Leo L. Beranek. Acoustics. McGraw-Hill, 1954.

H. J. Carlin and A. B. Giordano. Network theory, an introduction to reciprocal and nonreciprocal circuits. Englewood Cliffs NJ, 1964.

Mark Dodd, Wolfgang Klippel, and Jack Oclee-Brown. Voice coil impedance as a function of frequency and displacement. 102004.

Frederick V. Hunt. Electroacoustics: The analysis of transduction and its historical background. Harvard University Press, Harvard University, Massachusetts, 1954.

Joe Jensen, Finn T. Agerkvist, and James M. Harte. Nonlinear time-domain modeling of balanced-armature receivers. J. Audio Eng. Soc, 59(3):91-101, 2011.

Douglas H. Keefe. Acoustical wave propagation in cylindrical ducts: Transmission line parameter approximations for isothermal and nonisothermal boundary conditions. Journal of the Acoustical Society of America, 75(1):58-62, 1984.

Mead C. Killion. Elmer Victor Carlson: A lifetime of achievement. The Bulletin of the American Auditory Society, 17 (1):10-21, 1992.

E.M. McMillan. Violation of the reciprocity theorem in linear passive electromechanical system. J. Acoust. Soc. Am., 18:344-347, 1946.

Arnold Sommerfeld. Electrodynamics. Academic Press, 1964. 
B.D.H. Tellegen. The gyrator, a new electric network element. Philips Res. Rep t., 3:81-101, 1948.

Knud Thorborg, Andrew D. Unruh, and Christopher J. Struck. A model of loudspeaker driver impedance incorporating eddy currents in the pole structure. J. Audio Eng. Soc., 2007.

M. E. Van Valkenburg. Network Analysis. Prentice-Hall, Englewood Cliffs, NJ, 2nd edition, 1964.

J. Vanderkooy. A model of loudspeaker driver impedance incorporating eddy currents in the pole structure. J. Audio Eng. Soc., 37(3):119-128, 1989.

Daniel M. Warren and Janice L. LoPresti. A ladder network impedance model for lossy wave phenomena. The Journal of the Acoustical Society of America, (abst) 119(5):3377, 2006.

R.E. Weece and Jont Allen. A clinical method for calibration of bone conduction transducers to measure the mastoid impedance. Hearing Research, 263:216-223, 2010.

R. L. Wegel. Theory of magneto-mechanical systems as applied to telephone receivers and similar structures. Journal of the American Institute of Electrical Engineers, 40:791-802, 1921. 